\title{
Critical role of endothelial CXCR2 in LPS- induced neutrophil migration into the lung
}

\author{
Jörg Reutershan, ${ }^{1,2}$ Margaret A. Morris,, 1,3 Tracy L. Burcin,, David F. Smith,, Daniel Chang, ${ }^{1}$
} Mary S. Saprito, ${ }^{1}$ and Klaus Ley ${ }^{1,3,4}$

\begin{abstract}
${ }^{1}$ Robert M. Berne Cardiovascular Research Center, University of Virginia, Charlottesville, Virginia, USA. 2Department of Anesthesiology and Intensive Care Medicine, University of Tübingen, Tübingen, Germany. ${ }^{3}$ Department of Biomedical Engineering and ${ }^{4}$ Department of Physiology and Biological Physics, University of Virginia, Charlottesville, Virginia, USA.
\end{abstract}

\begin{abstract}
In models of acute lung injury, CXC chemokine receptor 2 (CXCR2) mediates migration of polymorphonuclear leukocytes (PMNs) into the lung. Since CXCR2 ligands, including CXCL1 and CXCL2/3, are chemotactic for PMNs, CXCR2 is thought to recruit PMNs by inducing chemotactic migration. In a model of PMN recruitment to the lung, aerosolized bacterial LPS inhalation induced PMN recruitment to the lung in wild-type mice, but not in littermate $C X C R 2^{-/-}$mice. Surprisingly, lethally irradiated wild-type mice reconstituted with $C X C R 2^{-/-} \mathrm{BM}$ still showed about 50\% PMN recruitment into bronchoalveolar lavage fluid and into lung interstitium, but $\mathrm{CXCR2}^{-/-}$mice reconstituted with $\mathrm{CXCR2}{ }^{-/-} \mathrm{BM}$ showed no PMN recruitment. Conversely, $\mathrm{CXCR2} 2^{-/-}$mice reconstituted with wild-type BM showed a surprisingly large defect in PMN recruitment, inconsistent with a role of CXCR2 on PMNs alone. Cell culture, immunohistochemistry, flow cytometry, and real-time RT-PCR were used to show expression of CXCR2 on pulmonary endothelial and bronchial epithelial cells. The LPS-induced increase in lung microvascular permeability as measured by Evans blue extravasation required CXCR2 on nonhematopoietic cells. Our data revealed what we believe to be a previously unrecognized role of endothelial and epithelial CXCR2 in LPS-induced PMN recruitment and lung injury.
\end{abstract}

\section{Introduction}

Acute lung injury (ALI) and acute respiratory distress syndrome (ARDS) are clinical syndromes characterized by an excessive inflammatory response to both pulmonary and extrapulmonary stimuli that ultimately leads to a disruption of alveolar-capillary integrity with severe consequences for pulmonary gas exchange. Both ALI and ARDS are still associated with a high mortality, and a specific therapy is not available (1).

Recruitment of neutrophils (polymorphonuclear leukocytes; PMNs) into the lung is a key event in the early development of ALI and ARDS, as previously demonstrated in neutropenic animals and humans $(2,3)$. PMN recruitment into the lung occurs in a cascade-like sequence of activation, sequestration in pulmonary vessels, and transendothelial (from blood to interstitium) and transepithelial (from interstitium to alveolar airspace) migration (4). Although interactions between leukocytes and endothelium have been well characterized in the systemic microcirculation, molecular requirements in the lung are not completely understood. Depending on the injury model and stimulus, adhesion molecules on leukocytes and endothelium may or may not be involved due to unique properties of the pulmonary microcirculation (5). Each migration step is regulated by distinct molecules (6), and the importance of investigating discrete steps of PMN migration in the lung has been emphasized (7).

CXCR2 is a 7-transmembrane $\mathrm{G}$ protein-coupled receptor that is activated by CXC chemokines containing the ELR (GluLeu-Arg) motif, including murine CXCL1 (keratinocyte-derived

Nonstandard abbreviations used: ALI, acute lung injury; ARDS, acute respiratory distress syndrome; BMT, BM transplantation; CXCR, CXC chemokine receptor; GR-1, granulocyte-1.

Conflict of interest: The authors have declared that no conflict of interest exists. Citation for this article: J. Clin. Invest. doi:10.1172/JCI27009. chemokine; KC) and CXCL2/3 (macrophage inflammatory protein 2; MIP-2) (8). Both chemokines are functional homologs of human CXCL8 (IL-8), which has been associated with the development and outcome of ARDS $(9,10)$. Upon activation, CXCR2 is phosphorylated, leading to receptor desensitization and internalization, intracellular removal of the ligand, and finally, degradation or re-expression of the receptor on the cell membrane (11). CXCR2 is expressed on PMNs, where its activation induces a variety of cell responses including degranulation, respiratory burst, phagocytosis, directed cell movement, integrin activation, and transmigration (12-14). Among nonhematopoietic cells, CXCR2 expression has been demonstrated in pulmonary endothelial cells (15), where it was associated with angiogenetic activity in lung tumors (16) and fibroproliferative processes (17), but its involvement in PMN migration was not tested. Both knockout and receptor-blocking strategies have revealed that CXCR2 is a pivotal mediator of PMN recruitment in lung injury (18-20). Although CXCR2 expressed by nonhematopoietic cells has been discussed (21), the role of endothelial and possibly epithelial CXCR2 in acute lung inflammation remains to be defined.

In the present study, we investigated the role of leukocytic, endothelial, and epithelial CXCR2 in a murine model of LPSinduced lung injury by creating chimeric mice expressing CXCR2 on either hematopoietic or nonhematopoietic cells. Our data showed that endothelial/epithelial CXCR2 was a major contributor to LPS-induced PMN recruitment and lung damage.

\section{Results}

CXCR2 mediates LPS-induced PMN recruitment into bronchoalveolar lavage. In $\mathrm{CXCR2}^{+/+}$mice, LPS inhalation (12 hours) induced significant PMN recruitment $\left(3.6 \pm 1.1 \times 10^{6}\right.$ cells $)$ into bronchoalveolar lavage (BAL; Figure 1). As expected (21), CXCR2-- mice were completely protected from PMN influx into the alveolar airspace. $C X C R 2^{+/-}$mice 


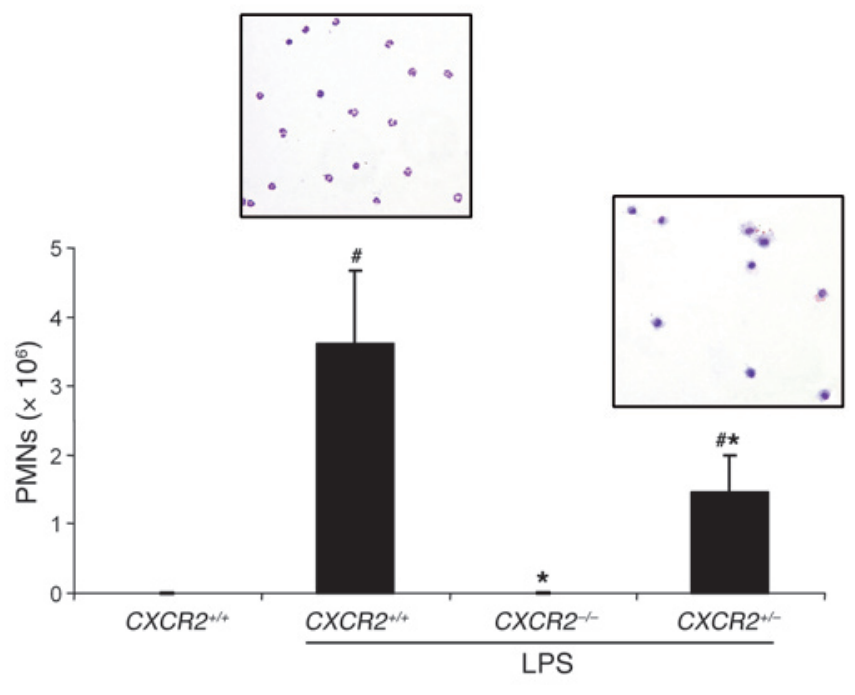

exhibited significant, but reduced, PMN migration upon LPS stimulation $\left(1.5 \pm 0.5 \times 10^{6}\right.$ cells $)$. This suggests that the expression level of CXCR2 is rate limiting under (patho)physiological conditions. Cytospins of BAL fluid confirmed PMN as the predominant cell type in $\mathrm{CXCR}^{+/+}$mice after LPS stimulation (Figure 1). In contrast, BAL fluid of $\mathrm{CXCR} 2^{-/-}$mice was dominated by (resident) alveolar macrophages. Although total leukocyte counts did not differ between CXCR2 $2^{+/+}$and $C X C R 2^{-/-}$mice, CXCR2 $2^{-/-}$mice exhibited significantly greater PMN counts (Table 1), as observed previously (22), which makes the observed reduction in migration even more striking.

CXCR2 mediates transendothelial PMN migration into the lung interstitium. After we found near-complete abolishment of PMN migration into the alveolar airspace in CXCR2 $2^{--}$mice, we investigated which of the trafficking steps (vascular accumulation, transendothelial migration, and transepithelial migration) was mediated by CXCR2. We assessed PMN counts in the different lung compartments using flow cytometry. PMNs in the lung homogenate were identified by their typical appearance in the forward scatter-side scatter (Figure 2A) and their expression of CD45 and CD11b. Granulocyte-1 (GR-1, also known as Ly6C/G) was injected intravenously and served as a marker for intravascular PMNs (CD11 b ${ }^{+}$GR$1^{+}$). Accordingly, CD $11 \mathrm{~b}^{+} \mathrm{GR}-1^{-}$cells represented interstitial PMNs after alveolar PMNs had been removed. In control CXCR2 ${ }^{+/+}$mice (saline inhalation), PMNs were detected in the pulmonary vasculature, consistent with the concept of a physiologically marginated pool (23). Almost no PMNs were observed in the interstitial space (Figure $2 \mathrm{~B})$. LPS inhalation induced significant $(P<0.001)$ PMN migration into the interstitium of $C X C R 2^{+/+}$mice $(69 \%$ of all pulmonary PMNs; Figure 2C). In CXCR2 $2^{--}$mice, LPS-induced accumulation of PMNs in the pulmonary vasculature was not reduced. However, recruited PMNs did not migrate into the lung interstitium (Figure 2D), suggesting a critical role for CXCR2 in transendothelial migration.

In vitro chemotaxis. Next we investigated whether altered chemotactic activity in BAL from CXCR2 $2^{-/}$mice contributed to defective PMN migration. LPS-exposed BAL, CXCL1, and CXCL2/3 were used in an in vitro transmigration assay. A 96-well chemotaxis system was loaded with chemoattractants, and BM-derived PMNs from either $\mathrm{CXCR} 2^{+/+}$or $C X C R 2^{-/-}$mice were placed on top of the filter above each well. As expected, LPS-exposed BAL induced a

\section{Figure 1}

LPS inhalation caused a significant recruitment of PMN into the BAL. In CXCR2 $2^{-1-}$ mice, PMN recruitment was completely abolished. In mice heterozygous for the CXCR2 allele $\left(C X C R 2^{+/-}\right)$, a reduction of $\sim 50 \%$ was observed. Data are mean $\pm \mathrm{SD}$ of $n=6$ mice in each group. Cytospins of LPS-exposed BAL in CXCR2 $2^{+/+}$and $C X C R 2^{-/-}$mice are shown above the corresponding bars. ${ }^{*} P<0.05$ versus $C X C R 2^{+/+}$; $P<0.05$ versus no LPS.

significant migration of PMNs from $\mathrm{CXCR} 2^{+/+}$mice (Figure 3). Furthermore, both CXCL1 and CXCL2/3 exhibited chemotactic activity. PMNs from $\mathrm{CXCR} 2^{-/-}$mice did not migrate toward CXCL1 or CXCL2/3. Surprisingly, PMNs from CXCR2 $2^{-/}$mice migrated to LPS-exposed BAL from CXCR2 $2^{-/-}$mice (Figure 3 ) and $\mathrm{CXCR}^{+/+}$ mice (data not shown), suggesting the contribution of other, CXCR2-independent chemoattractants. However, CXCR2-independent migration seemed to be negligible in vivo. This led us to hypothesize that endothelial CXCR2, which was not a factor of the in vitro assay, might be required for PMN migration in mice.

PMN trafficking in chimeric mice. To investigate the role of endothelial CXCR2, we created chimeras and controls by transferring $\mathrm{BM}$ between $\mathrm{CXCR2} 2^{+/}$and $\mathrm{CXCR2} 2^{-/-}$mice. Animals were used for experiments 6 weeks after BM transplantation (BMT). Complete reconstitution was confirmed by real-time RT-PCR (Figure 4A). In all groups, mice were assessed 12 hours after LPS inhalation. PMNs were detected in all lung compartments as described above. Consistent with the findings in CXCR2 $2^{-/-}$mice, $\mathrm{CXCR2^{-/- }}$ mice

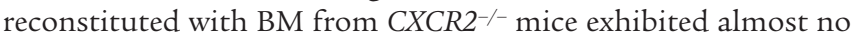
LPS-induced transendothelial PMN migration and no PMNs in BAL (Figure 4B). When $C X C R 2^{+/+}$mice were reconstituted with $\mathrm{BM}$

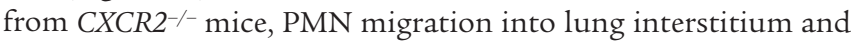
alveolar airspace were reduced by approximately $50 \%$, confirming that CXCR2 on hematopoietic cells was involved in PMN migration. Surprisingly, a very similar reduction was seen when CXCR2-/mice were reconstituted with $\mathrm{BM}$ from $\mathrm{CXCR} 2^{+/+}$mice, suggesting a critical role for CXCR2 on nonhematopoietic cells. Intravascular PMN accumulation did not differ among the groups.

Endothelial CXCR2 expression. To investigate the expression of CXCR2 on pulmonary endothelial cells, RNA was extracted from isolated lung endothelial cells, and the amount of CXCR2 mRNA was determined and normalized to GAPDH. Pulmonary endothelial cells expressed CXCR2 mRNA (Figure 5). CXCR2 mRNA was also detected in BAL from $C X C R 2^{+/+}$, but not $\mathrm{CXCR}^{-{ }^{--}}$, mice. In $\mathrm{CXCR2}^{+/+}$mice reconstituted with $\mathrm{BM}$ from $\mathrm{CXCR} 2^{-/-}$mice, low levels of CXCR2 mRNA were found, suggesting that most CXCR2

\section{Table 1}

Baseline cell counts

\begin{tabular}{lccc}
\hline & & & \\
& CXCR2 ${ }^{+/+}$ & CXCR2-- & $\boldsymbol{P}$ \\
Leukocytes & $5.5 \pm 1.6$ & $5.9 \pm 1.4$ & 0.72 \\
PMNs & $1.0 \pm 0.2$ & $3.7 \pm 1.4$ & $<0.05$ \\
Lymphocytes & $4.3 \pm 1.6$ & $2.2 \pm 0.4$ & $<0.05$ \\
Monocytes & $0.2 \pm 0.1$ & $0.4 \pm 0.2$ & 0.13 \\
PMNs (\%) & $18.4 \pm 4.1$ & $58.5 \pm 10.8$ & $<0.05$ \\
Lymphocytes (\%) & $77.0 \pm 6.0$ & $35.2 \pm 6.0$ & $<0.05$ \\
Monocytes (\%) & $4.4 \pm 2.3$ & $7.3 \pm 3.8$ & 0.25
\end{tabular}

Baseline differential cell counts were performed in $C X C R 2^{+/+}$and

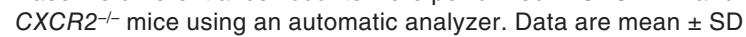
of 4 samples. 
A
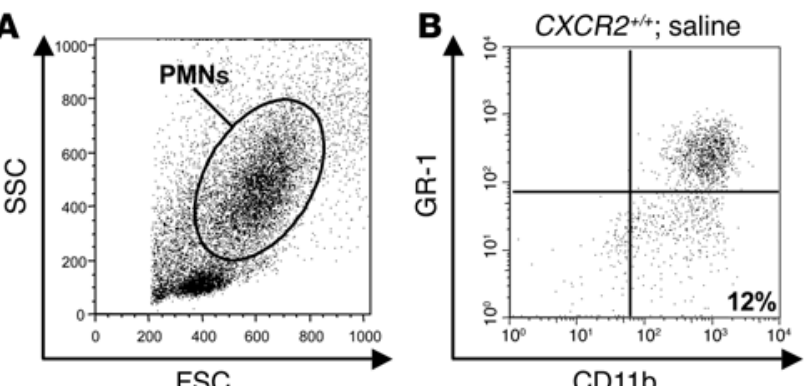

C

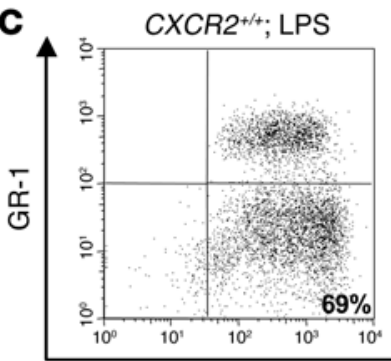

D

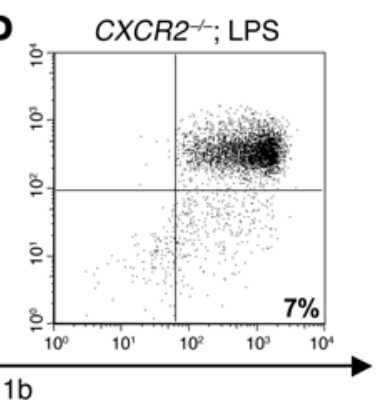

mRNA in BAL came from hematopoietic cells. Consistent with this interpretation, $\mathrm{CXCR2^{-/- }}$ mice reconstituted with $\mathrm{CXCR} 2^{+/+} \mathrm{BM}$ showed almost normal CXCR2 expression in BAL cells.

Next, CXCR2 expression was investigated by immunohistochemistry. In $\mathrm{CXCR2^{+/+ }}$ mice, strong endothelial expression of CXCR2 protein was found in large and small pulmonary vessels as well as within alveolar septal walls (Figure 6A, arrow). Interestingly, a strong CXCR2 expression was also detected along the epithelial layer of larger airways (Figure 6C). In contrast, CXCR2-/- did not show any CXCR2 expression (Figure 6, B and D)

In lung homogenate, leukocytes $\left(\mathrm{CD} 45^{+}\right)$and nonleukocytes $\left(\mathrm{CD} 45^{-}\right)$were analyzed separately for their expression of CXCR2 by flow cytometry (Figure $7 \mathrm{~B}$ ). Among $\mathrm{CD}_{4} 5^{+}$cells, only GR-1 $1^{\text {high }}$ PMNs expressed detectable CXCR2 (Figure 7C). The majority of endothelial cells (CD45-CD31 ${ }^{+}$), but not all, exhibited CXCR2 expression (Figure 7A). In addition, CXCR2 was also found on a sizeable population of $\mathrm{CD} 45^{-} \mathrm{CD} 31^{-}$cells, probably representing bronchial epithelial cells.

Pulmonary microvascular permeability. In addition to PMN infiltration, vascular leakage is most critical in ALI. We therefore tested the role of endothelial and leukocytic CXCR2 in the LPS-induced increase in microvascular permeability. In $\mathrm{CXCR}^{+/+}$ mice, LPS inhalation caused a significant increase in Evans blue leakage into the lung (Figure 8). This increase was absent in CXCR2 $2^{-/-}$mice as well as in CXCR2 $2^{-/-}$mice after reconstitution with $\mathrm{BM}$ from $\mathrm{CXCR} 2^{+/+}$mice. In contrast, $\mathrm{CXCR} 2^{+/+}$mice reconstituted with BM from $C X C R 2^{-/-}$mice exhibited almost normal LPS-induced protein leakage, suggesting that endothelial CXCR2 is critical for LPS-induced protein leakage in lung injury.

Cytoskeletal remodeling. To test whether endothelial CXCR2 activation induces cytoskeletal rearrangements of endothelial cells, we stained for F-actin in isolated pulmonary endothelial cells (Figure 9). Stimulation with CXCL1 induced a marked increase in F-actin-positive stress fibers as early as 5 minutes after activation. Stress fibers were most pronounced at cell-cell borders. This effect was dose dependent. After 120 minutes, most endothelial cells

\section{Figure 2}

Flow cytometry of lung homogenate. (A) PMNs were identified by their typical appearance in the forward scatter (FSC) and side scatter (SSC) and their expression of CD45 and CD11b (data not shown). GR-1 was injected intravenously to distinguish between intravascular and extravascular (i.e., interstitial after removal of BAL) PMNs. (B) In control animals (saline inhalation), $88 \%$ of PMNs were found intravascularly. (C) LPS caused transendothelial migration into the interstitium, as demonstrated by the appearance of CD11+GR-1- PMNs. (D) In contrast, transendothelial migration was suppressed in $\mathrm{CXCR} 2^{-/-}$mice.

appeared in a polygonal, retracted shape. No effects were observed in untreated cells (data not shown). This confirms the potential of endothelial CXCR2 to mediate vascular permeability.

\section{Discussion}

In a murine model of ALI, we characterized the involvement of CXCR2 in the different migration steps of PMN and in vascular leakage. We found that CXCR2 is crucial for transendothelial and transepithelial migration of PMNs, but not for their accumulation in the pulmonary vasculature in response to aerosolized LPS. In addition to PMNs responding to a chemotactic gradient generated by CXCR2 ligands at the site of inflammation, we identified what we believe to be a previously unrecognized role for endothelial CXCR2 in mediating endotoxin-induced PMN migration and protein leakage.

CXCR2 plays a major role in experimental ALI. CXCR2 $2^{-/-}$mice were previously shown to be protected from hyperoxia-induced PMN infiltration, lung edema, and vascular protein leakage (18). In addition, CXCR2 expression in the lung was shown to increase with high-peak pressure ventilation, and mice were protected from ventilator-induced lung damages by using anti-CXCR2 strategies (21). It was previously determined that the beneficial effects of ventilation with lower tidal volumes observed in ARDS patients (24) might be attributed to preventing CXCR2-dependent leukocyte recruitment. In both studies $(18,21)$, indicators of lung injury were restored almost to baseline.

In our study, $C X C R 2^{-/-}$mice were completely protected from endotoxin-induced PMN migration and vascular protein leakage. Based on previous studies, this was expected, but it is still remark-

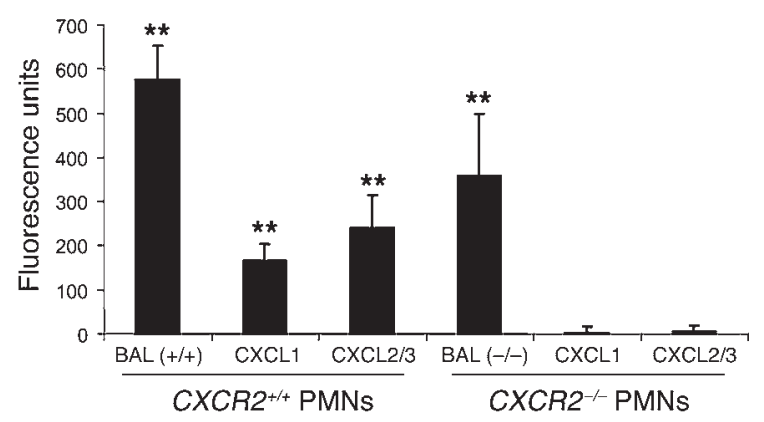

Figure 3

Chemotactic activity of LPS-exposed BAL from $C X C R 2^{+/+}$mice and the 2 critical CXCR2 ligands, CXCL1 and CXCL2/3, was assessed in an in vitro transmigration assay. PMNs isolated from $C X C R 2^{+/+}$mice showed significant migration toward LPS-exposed BAL, CXCL1, and $C X C L 2 / 3$. PMNs from $C X C R 2^{-/-}$mice did not migrate toward the CXCR2 ligands, but still migrated toward LPS-exposed BAL from $\mathrm{CXCR}^{-1-}$ mice. Data are mean $\pm \mathrm{SD}$ of 3 experiments. ${ }^{* \star} P<0.001$ versus negative control (medium only). 

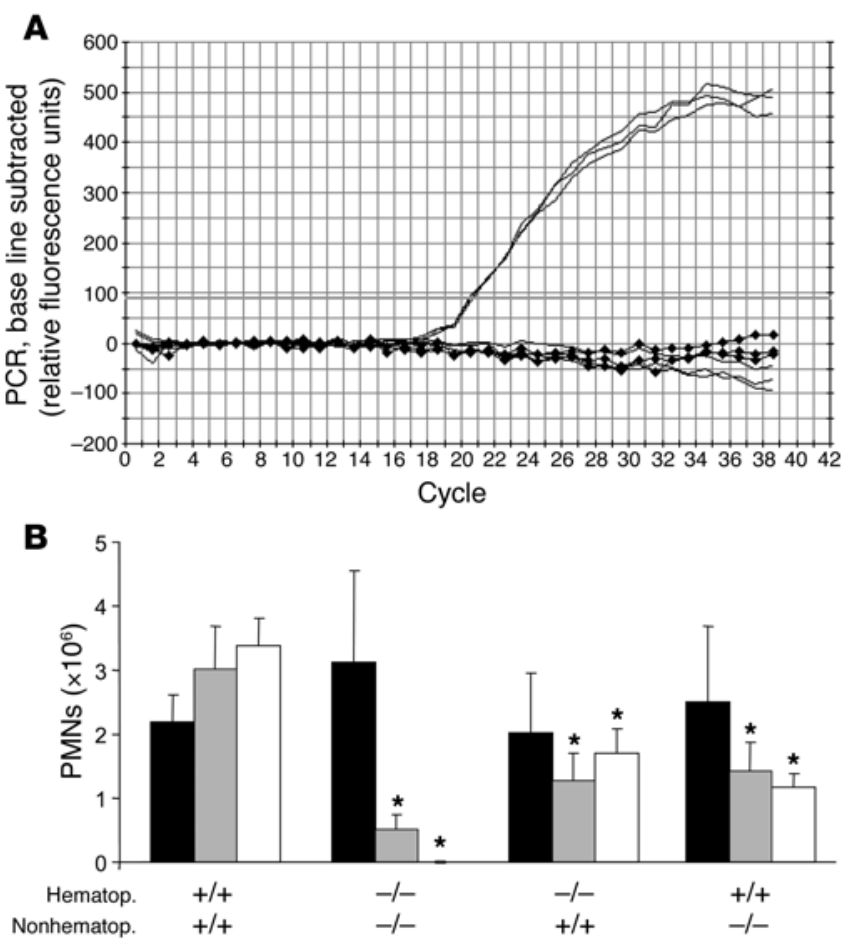

able in that many other strategies, such as antiadhesive approaches, inhibit PMN migration into the lung only partially, if at all (25-27). To our knowledge, no model of lung injury has yet been demonstrated to be CXCR2 independent, confirming the pivotal role of this chemokine receptor.

Recently, a murine CXCR1-like receptor has been reported by 2 independent groups $(28,29)$. This receptor has been confirmed by RT-PCR and Northern blot. However, in both studies, no receptor protein was detected. Activation with CXCL1, CXCL2/3, or CXCL8 had no effect on CXCR1-expressing cells. Further investigations are needed to confirm the presence of this receptor as well as its biological impact. Our studies as well as others' $(18,21)$ showed that PMN migration was reduced almost to baseline in CXCR2 $2^{-/-}$mice, suggesting that the contribution of CXCR1 might not be important.

The current concept of PMN recruitment into the lung includes the production of chemoattractants, for instance the chemokines CXCL1 and CXCL2/3, by stimulated alveolar macrophages or other cells in the alveolar compartment (30). This is thought to generate a chemotactic gradient attracting CXCR2-expressing leukocytes to the lung. Consistent with this idea, LPS-exposed BAL exhibited strong chemotactic activity. This was most pronounced when PMNs from $C X C R 2^{+/+}$mice were allowed to migrate toward BAL from $C X C R 2^{+/+}$mice. In an endothelial cell-free chemotaxis

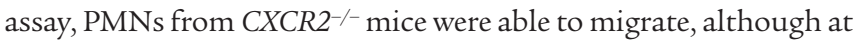
a reduced rate, toward LPS-exposed BAL from CXCR2 $2^{-1-}$ mice. This implicates the presence of a CXCR2-independent chemotactic pathway. In a previous study, incubation of human PMNs with a CXCL8 receptor inhibitor was shown to reduce CXCL8-, but not fMLP-, PAF-, LTB $_{4-}$, or C5a-induced chemotaxis $(14,31)$.

Our in vivo data show that CXCR2-independent PMN migration was negligible. However, PMN accumulation in the pulmonary vasculature in vivo occurred even in the absence of CXCR2. This is in line with previous data showing that pertussis toxin,

\section{Figure 4}

LPS-induced PMN migration into different lung compartments. (A) Complete reconstitution of hematopoietic cells after BMT was confirmed by RT-PCR (whole blood). Solid lines, positive $\left(C X C R 2^{+/+}\right)$and

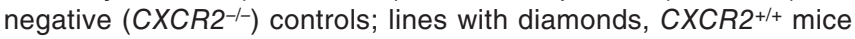

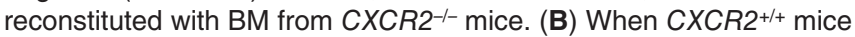

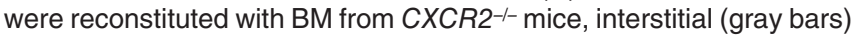
and BAL (white bars) PMN content was reduced by $40 \%$ and $50 \%$, respectively. When $C X C R 2^{-1-}$ mice were reconstituted with $\mathrm{BM}$ from CXCR2 $2^{++}$mice, the reduction was by $50 \%$ and $60 \%$, respectively. LPS-induced accumulation of PMN in the pulmonary vasculature (black bars) did not differ among the groups. CXCR2 $2^{+/+}$mice recon-

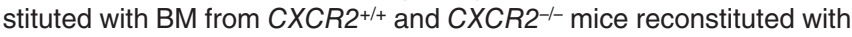

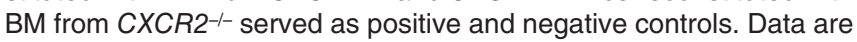
mean \pm SD of $n=4$ mice. ${ }^{*} P<0.05$ versus positive control.

an inhibitor of $\mathrm{G}_{\alpha i}$-dependent signaling, blocked LPS-induced PMN migration but not accumulation in the lung vasculature (4). It has been suggested that endothelial-derived or exogenous chemokines can be deposited on endothelial cells to mediate leukocyte arrest at inflammatory sites $(32,33)$. However, due to the unique architecture of the pulmonary microvasculature, chemokines may not be required for arrest to a firmly adherent state (34). Our data support this concept, as PMN accumulation was not reduced in CXCR2 $2^{-/-}$mice.

Surprisingly, PMN migration into the lung was suppressed when nonhematopoietic cells did not express CXCR2. Nonhematopoietic CXCR2 had a significant impact on both transendothelial and transepithelial migration, implicating a crucial role for endothelial and epithelial CXCR2. Endothelial expression of CXCR2 has previously been demonstrated in different human tissues (15). Belperio et al. demonstrated that endothelial CXCR2 mediated chemokineinduced angiogenesis in a model of lung cancer (16) and bronchiolitis obliterans syndrome (17). Anti-CXCR2 strategies were able to reduce injury in both models, but the role of endothelial and epithelial CXCR2 in PMN migration was unexpected. In our studies, CXCR2 was detected on two-thirds of all endothelial

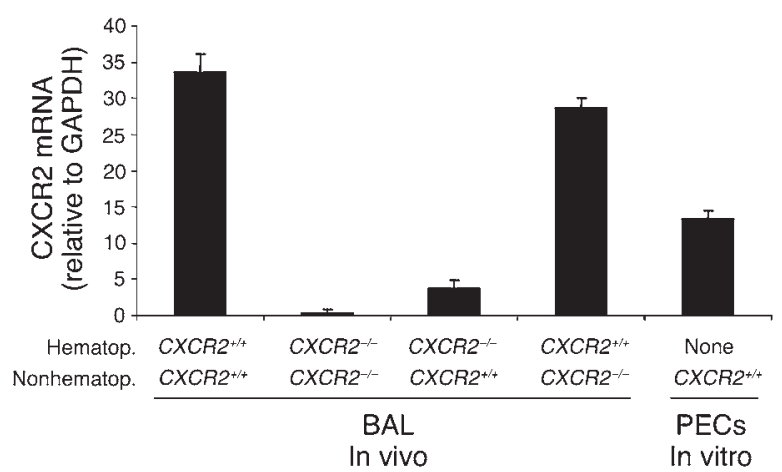

Figure 5

CXCR2 mRNA expression in pulmonary endothelial cells (PECs) and $\mathrm{BAL}$. Isolated pulmonary endothelial cells exhibited readily detectable amounts of CXCR2 mRNA. BAL from CXCR2 ${ }^{+/+}$also showed CXCR2 mRNA expression, but negligible $C X C R 2$ mRNA was found in the BAL of CXCR2 $2^{-1-}$ mice. In $\mathrm{CXCR} 2^{+/+}$mice reconstituted with $\mathrm{BM}$ from $\mathrm{CXCR} 2^{-/-}$ mice, low levels of CXCR2 mRNA were detected, most likely derived from contamination with epithelial cells. CXCR2 mRNA expression was almost normal when $C X C R 2^{-/-}$mice were reconstituted with $\mathrm{BM}$ from $C X C R 2^{+/+}$mice. CXCR2 mRNA levels were normalized to GAPDH and presented as mean $\pm \mathrm{SD}$ of $n=3$ samples. 

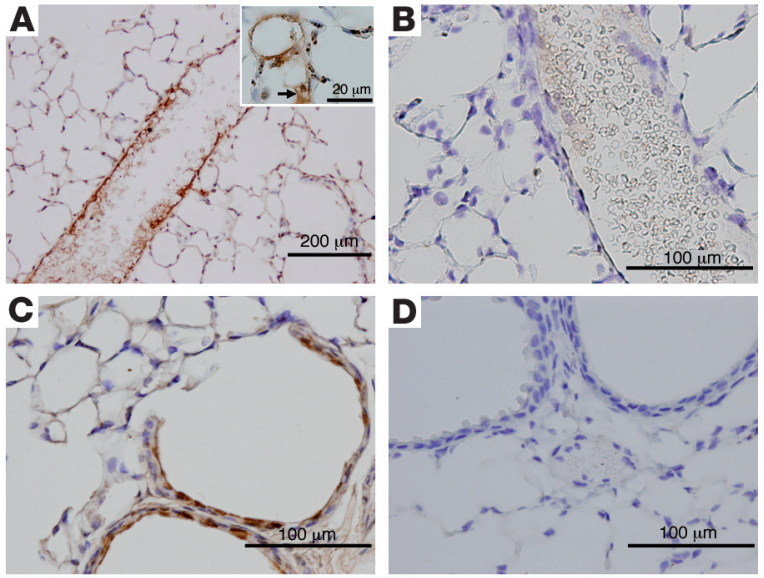

\section{Figure 6}

CXCR2 protein expression shown by immunohistochemistry. (A) Lungs from $\mathrm{CXCR} 2^{+/+}$mice demonstrated CXCR2 expression among large vessels, small vessels (insert), and alveolar septal walls (arrow) and among the epithelial layer of large airways (C). No CXCR2 was detected in lungs from $C X C R 2^{-1-}$ mice (B and $\left.\mathbf{D}\right)$.

cells (Figure 7A). Our present data did not allow us to identify the origin of CXCR2-expressing endothelial cells. As shown by immunohistochemistry, CXCR2 was detected on conduit vessels as well as within septal walls, where most capillaries reside. Our findings demonstrating that endothelial CXCR2 mediated PMN migration and vascular permeability are consistent with the presence of PMNs in capillaries, the most likely site of cell migration in the lung. However, this does not exclude PMN expression on endothelium of larger vessels, which might or might not be involved in cell migration and permeability.

We have demonstrated that activation of CXCR2 on endothelial cells resulted in rapid actin polymerization and formation of stress fibers, ultimately leading to a retraction of these cells. This is consistent with previous reports (35) and supports the potential of endothelial CXCR2 to mediate vascular permeability.

Interestingly, we also found strong expression of CXCR2 on epithelial cells. This is consistent with previous findings (30) demonstrating that type II pneumocytes in culture not only produce CXC chemokines but also their cognate receptor. Epithelial CXCR2, in addition to endothelial CXCR2, may contribute to cell retraction promoting recruitment of inflammatory cells and the formation of edema.

Clinical implications. At present, the absence of a specific therapy for ALI/ARDS results in substantial mortality, which has been decreasing only moderately over the past decades (36). In several studies, neutropenia has been shown to attenuate the extent of lung damage $(2,3)$, highlighting the pivotal role of PMN recruitment in the early development of the disease. The human CXCR2 (and CXCR1) ligand CXCL8 is produced in the BAL of patients with ARDS and largely accounts for its chemotactic activity (9). Moreover, CXCL8 levels in the BAL have been demonstrated to affect prognosis of ARDS. PMN recruitment to sites of inflammation is required to fight bacterial infections (37); thus an inhibition of PMN migration might have untoward consequences.
However, previous reports impressively demonstrated that genetic deletion or complete inhibition of CXCR2 improved survival in murine models of sepsis $(38,39)$, suggesting an immunomodulatory rather than immunosuppressive role of inhibiting CXCR2.

Our study showed a partial inhibition of PMN recruitment in mice heterozygous for the CXCR2 allele, suggesting a potential benefit of a pharmaceutical receptor-blocker capable of partially inhibiting its target. Furthermore, in humans, a specific CXCR2 inhibitor might allow CXCL8 to still act through CXCR1, which might be particularly important for chemokine-induced release of granule enzymes and superoxide generation (13). New types of specific CXCR2 inhibitors, including orally available reagents, are being developed (40, 41). Our data suggest that CXCR2 on nonhematopoietic cells may be a novel therapeutical target to control lung injury.

\section{Methods}

Mice. $\mathrm{CXCR2}^{+/-}$mice (22) on the Balb/c background were purchased from The Jackson Laboratory. Interbreeding of $C X C R 2^{+/-}$mice yielded $C X C R 2^{-/-}$, CXCR2 $2^{+/-}$, and $C X C R 2^{+/+}$pups at the expected Mendelian ratio. Wild-type littermates $\left(C X C R 2^{+/+}\right)$served as control animals. PCR of tail biopsies was performed to determine the CXCR2 genotype as previously described (22). All animal experiments were approved by the Animal Care and Use Committee of the University of Virginia. Mice were 8-12 weeks of age.

Generation of chimeric mice. Chimeric mice were generated by transferring $\mathrm{BM}$ as described previously (42). Briefly, recipient mice were lethally irradiated in 2 doses of 600 rad each (separated by 4 hours). BM from donor mice was harvested from both femora and tibiae, and approximately 5 million cells were injected intravenously into recipient mice. BMT was performed in 4 groups of mice: (a) BM from CXCR2 $2^{--}$into CXCR2 $2^{++}$(chimeric, expressing CXCR2 on nonhematopoietic cells only); (b) BM from

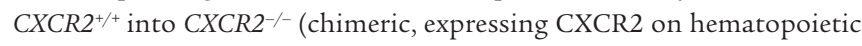
cells only); (c) BM from CXCR2 $2^{--}$into CXCR2 $2^{---}$; and (d) BM from $C X C R 2^{+/+}$ into $C X C R 2^{+/+}$. Mice in the latter 2 groups served as negative and positive controls for possible radiation effects. Chimeric mice were used for experiments 6 weeks after BMT, and complete reconstitution of the transferred BM was confirmed by real-time RT-PCR (primers: forward, AGCAAACACСТСТАСТАСССТCTA; reverse, GGGCTGCATCAATTCAAATACCA; probe, ACCTAAACGGTGGCCCCTCGGGG).

Murine model of $A L I$. We used an endotoxin inhalation approach to induce pulmonary PMN migration (4). Up to 4 mice were exposed to aerosolized LPS in a custom-built cylindrical chamber $(20 \mathrm{~cm} \times 9 \mathrm{~cm})$ connected to an
A
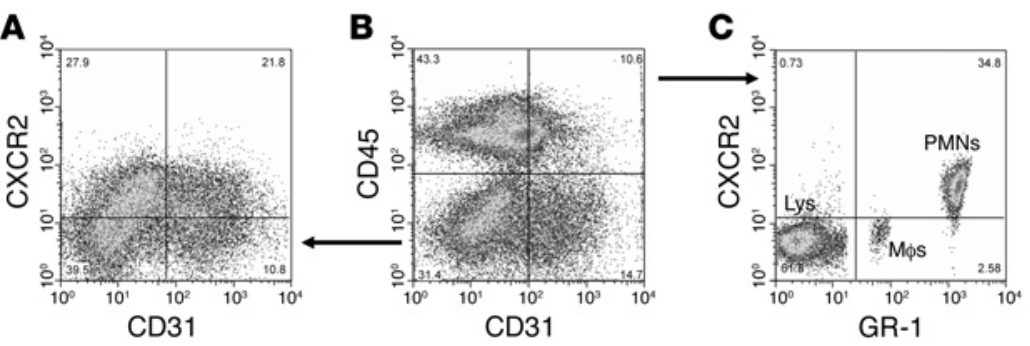

Figure 7

CXCR2 expression in lung homogenate. (B) Leukocytes (CD45 ${ }^{+}$and nonleukocytes (CD45-) were analyzed separately for their expression of CXCR2 (shown in $\mathbf{C}$ and A, respectively). (A) Among all CD45- cells, endothelial cells were identified by their expression of CD31. The majority of endothelial cells ( 67\%) expressed CXCR2. In addition, CXCR2 was found in a large population of CD31- cells. (C) Among all leukocytes $\left(C D 45^{+}\right)$, only PMNs (GR-1 $\left.{ }^{\text {high }}\right)$ exhibited CXCR2 surface expression. Neither monocytes/macrophages (M $\phi s ;$ GR-1 $1^{\text {intermediate }}$ ) nor most lymphocytes (Lys; GR-1 $\left.{ }^{\text {negative}}\right)$ showed detectable CXCR2 expression. 


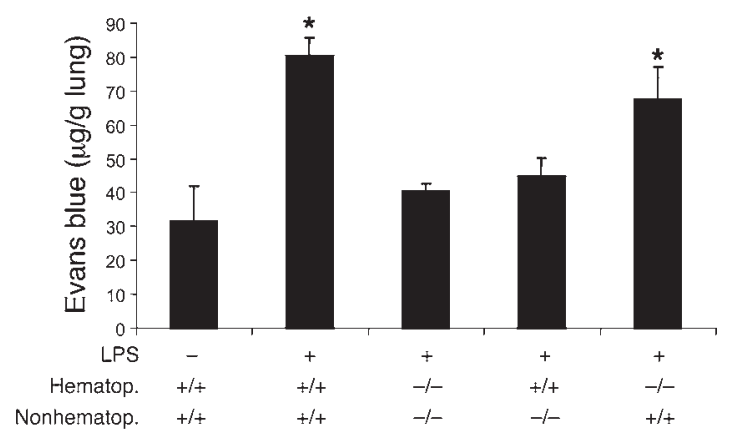

Figure 8

Pulmonary microvascular permeability was assessed in $C X C R 2^{+/+}$, CXCR2 $2^{--}$, and chimeric mice. In CXCR2 ${ }^{-/-}$mice and in $C X C R 2^{-/-}$mice reconstituted with $\mathrm{BM}$ from $C X C R 2^{+/+}$mice, LPS-induced protein leakage was significantly reduced compared with $C X C R 2^{+++}$mice. In contrast, no

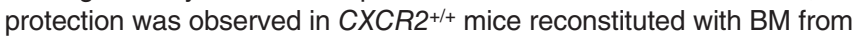
CXCR2 $2^{-1-}$. ${ }^{*} P<0.05$ versus $C X C R 2^{+/+}$mice. Data are mean $\pm \mathrm{SD}$ of $n=4$ mice.

air nebulizer (MicroAir; Omron Healthcare Inc.). The outlet of the chamber was connected to a vacuum pump, and a constant flow rate of $15 \mathrm{ml} /$ min was ensured by a flow meter (Gilmont Instruments). LPS from Salmonella enteritidis (Sigma-Aldrich) was dissolved in $0.9 \%$ saline $(500 \mu \mathrm{g} / \mathrm{ml})$, and mice were allowed to inhale LPS for 30 minutes. As previously shown by our laboratory, this results in a time-dependent PMN recruitment into all compartments of the lung with a peak between 12 and 24 hours (4). Control mice were exposed to saline aerosol.

Assessment of PMN trafficking in the lung. PMN recruitment into the 3 compartments of the lung (pulmonary vasculature, interstitium, and alveolar airspace) was assessed 12 hours after LPS exposure. Mice were anesthetized with an intraperitoneal injection of ketamine $(125 \mathrm{mg} / \mathrm{kg}$; Sanofi Winthrop Pharmaceuticals Inc.), xylazine (12.5 mg/kg; Phoenix Scientific Inc.) and atropine sulfate $(0.025 \mathrm{mg} / \mathrm{kg}$; Fujisawa). To determine PMN counts in the alveolar airspace, the ribcage was opened through a midline incision, and the trachea was cannulated (22 GA Insyte; BD) and $5 \times 1 \mathrm{ml}$ of cold PBS was infused and withdrawn. BAL fluid was centrifuged for 5 minutes at $300 \mathrm{~g}$. The pellet was resuspended in $1 \mathrm{ml}$ PBS with $1 \%$ BSA and $0.1 \%$ sodium azide, and a 10- $\mu$ l aliquot was used to count cells in a hemocytometer (Reichert Inc.). Cell viability was determined by Trypan Blue (Sigma-Aldrich) exclusion.

Percentage fraction of PMNs in BAL was determined by flow cytometry (FACSCalibur; BD). PMNs were identified by their typical appearance in the forward/side scatter and their expression of CD45 (clone 30-F11), CD11b (clone M1/70), and GR-1 (clone RB6-8C5). Appropriate isotype controls (rat IgG2b) were used to compensate for nonspecific antibody binding. All antibodies were purchased from BD Biosciences - Pharmingen except the anti-mouse GR-1 antibody, which was purified from supernatant of the GR-1 hybridoma (ATCC) by the biomolecular facility of the University of Virginia and fluorescently labeled with an $\mathrm{F}(\mathrm{ab})$-based staining kit following the manufacturer's directions (Alexa Fluor 633; Invitrogen Corp.).

In order to track PMNs in the different lung compartments, we recently developed a flow cytometry-based method (4). Five minutes prior to euthanasia, Alexa 633-labeled GR-1 to murine PMNs was injected intravenously. We have previously shown that this results in a complete labeling of all intravascular, but not interstitial or alveolar, PMNs (4). After 5 minutes, mice were euthanized, and nonadherent PMN were removed from the pulmonary vasculature by flushing $10 \mathrm{ml}$ of $\mathrm{PBS}$ at $25 \mathrm{~cm} \mathrm{H}_{2} \mathrm{O}$ through the spontaneously beating right ventricle after cutting the inferior vena cava to allow exsanguination. BAL was performed as described above to retrieve alveolar PMNs. Then lungs were removed, minced, and digested with $125 \mathrm{U} / \mathrm{ml}$ collagenase type $\mathrm{XI}, 60 \mathrm{U} / \mathrm{ml}$ hyaluronidase type I-s, and $60 \mathrm{U} / \mathrm{ml}$ DNase 1 (all from Sigma-Aldrich) at $37^{\circ} \mathrm{C}$ for 30 minutes. Addition of excess unlabeled anti-GR-1 prevented possible binding of the injected antibody to extravascular PMNs. A cell suspension was made by passing the digested lungs through a $70-\mu \mathrm{m}$ cell strainer (BD Biosciences - Falcon). Erythrocytes were lysed, and remaining leukocytes were resuspended and counted. PMNs were then identified as described above, except that the expression of GR-1 was used to distinguish intravascular $\left(\mathrm{CD} 45^{+} \mathrm{CD} 11 \mathrm{~b}^{+} \mathrm{GR}-1^{+}\right)$from interstitial $\left(\mathrm{CD} 45^{+} \mathrm{CD} 11 \mathrm{~b}^{+} \mathrm{GR}-1^{-}\right)$PMNs, which were not reached by the injected antibody. To confirm the purity of PMNs within the different populations as defined by flow cytometry, we sorted both $\mathrm{CD} 45^{+} \mathrm{CD} 11 \mathrm{~b}^{+} \mathrm{GR}-1^{+}$and $\mathrm{CD} 45^{+} \mathrm{CD} 11 \mathrm{~b}^{+} \mathrm{GR}-1^{-}$cells within the appropriate forward/side scatter gate (FACSVantage; $\mathrm{BD}$ ) and characterized them morphologically by cytospin (Diff-Quick staining; IMEB Inc.).

Expression of CXCR2 in PMNs and pulmonary endothelial cells in the lung homogenate was determined by flow cytometry. Anti-CD31 (clone MEC 13.3; BD Biosciences - Pharmingen) was used to identify endothelial cells $\left(\mathrm{CD} 31^{+} \mathrm{CD} 45^{-}\right)$. CXCR2 expression was determined with a monoclonal rat anti-mouse CXCR2 antibody (R\&D Systems). Appropriate isotypes were used to set the FACS gates.

$P M N$ chemotaxis assay. Altered chemotactic activity in the BAL from CXCR2 $2^{-/-}$mice might affect PMN migration into the lung. To test this hypothesis, we performed an in vitro chemotaxis assay. BM from $C X C R 2^{+/+}$ and $\mathrm{CXCR2} 2^{--}$mice was harvested as described above, and PMNs were purified from a 3-layer Percoll gradient (78\%, 66\%, and 54\%) (43). Isolated PMNs were labeled with calcein AM (5 $\mu \mathrm{M}$; Invitrogen Corp.) and washed twice. A 96-well chemotaxis system (ChemoTx, 3- $\mu$ m filter pore size; Neuro

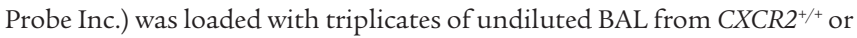
CXCR2 $2^{-/-}$mice (3 hours after LPS exposure), recombinant CXCL1 (250 ng/ $\mathrm{ml}$; PeproTech), or recombinant CXCL2/3 (250 ng/ml; PeproTech). This chemokine concentration was found to be optimal for PMN chemotaxis within a tested range of $10-1,000 \mathrm{ng} / \mathrm{ml}$ (data not shown). PMN suspension $\left(25 \mu l, 3.0 \times 10^{6} / \mathrm{ml}\right)$ was placed on top of the filter above each well, and the chamber was incubated for 1 hour at $37^{\circ} \mathrm{C}$ and $5 \% \mathrm{CO}_{2}$ (44). After incubation, nonmigrated cells were removed from the top of the filter, and fluorescence in the wells (excitation, $485 \mathrm{~nm}$; emission, $530 \mathrm{~nm}$ ) was measured with a multiwell fluorescence plate reader. Values were corrected for baseline fluorescence (medium only).

Isolation of pulmonary endothelial cells. Pulmonary endothelial cells were isolated using a positive immunomagnetic selection (modified from ref. 6). Lungs were removed, minced, and digested in $0.2 \%$ collagenase (SigmaAldrich). A single-cell suspension was prepared by passing the digested lungs through a $70-\mu \mathrm{m}$ cell strainer (BD Biosciences - Falcon), and cells were incubated with biotinylated anti-CD31 (Mec 13.3) at $4^{\circ} \mathrm{C}$ for 1 hour. Cells were then washed, and magnetic antibiotin nanoparticles (EasySep Biotin Selection Kit; StemCell Technologies) were added. After 60 minutes of incubation, unlabeled cells were poured off. Endothelial cells were cultured in gelatin-coated flasks until confluent and used for real-time PCR.

CXCR2 real-time PCR. Real-time RT-PCR was performed on samples from $C X C R 2^{+/+}$and $C X C R 2^{-/-}$mice to confirm complete reconstitution in mice undergoing BMT and assess CXCR2 mRNA expression in pulmonary endothelial cells. RT-PCR primers and Taqman probes were designed using Beacon Designer V4.0 software (PREMIER Biosoft International). Total RNA was isolated using RNeasy Mini-Kit (QIAGEN) with DNase treatment (RNase-Free DNase Kit; QIAGEN). Reverse transcription was performed using Omniscript RT Kit (QIAGEN) and oligo-dT primers. The primers were tested for specificity by standard PCR using CDNA made from both $C X C R 2^{+/+}$ and $C X C R 2^{--}$mRNA. This confirmed that wild-type samples produced the correct sized band, while no band was present in the knockout samples. 

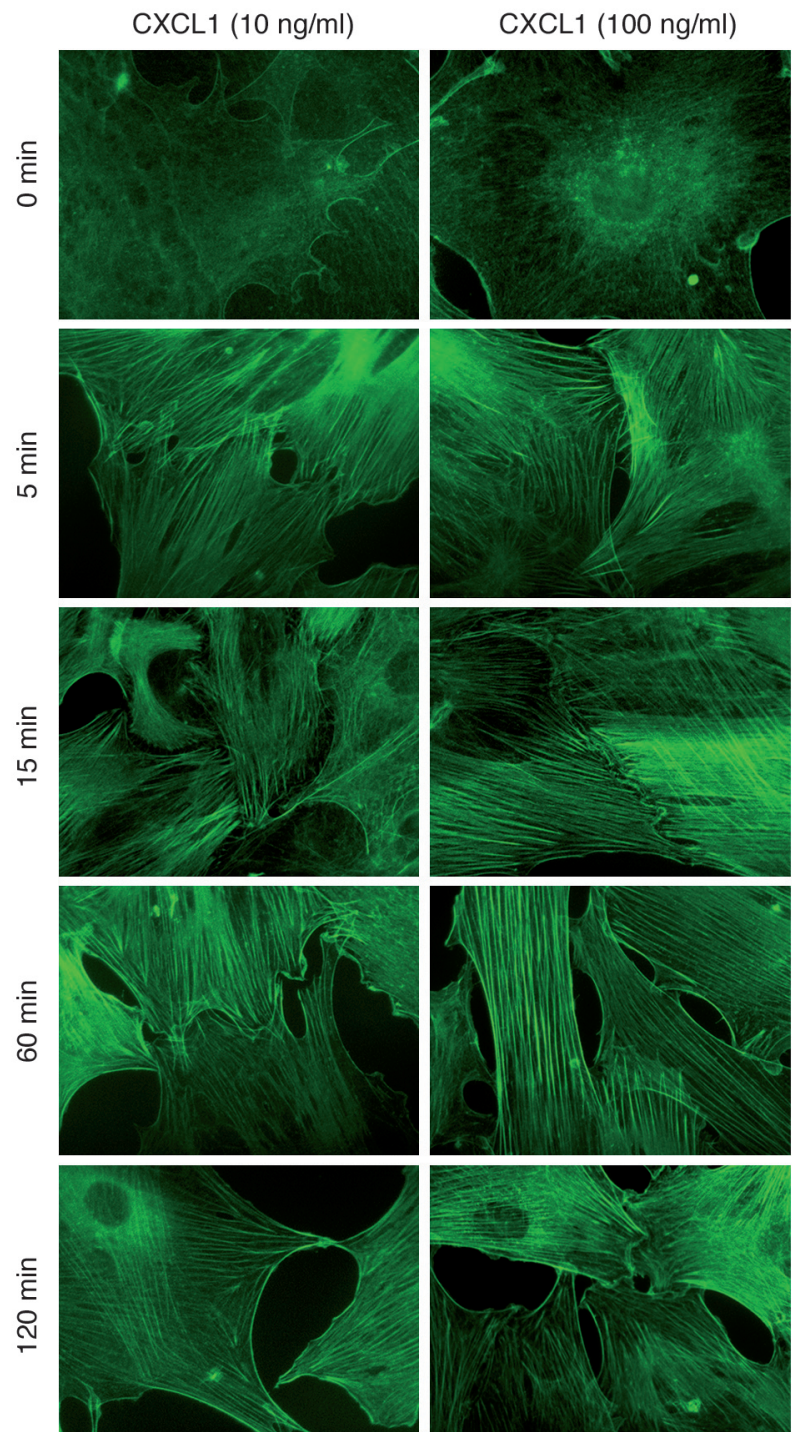

\section{Figure 9}

Endothelial cell response to CXCR2 activation was investigated by $\mathrm{F}$-actin localization. Isolated pulmonary endothelial cells were treated with 10 or $100 \mathrm{ng} / \mathrm{ml}$ of recombinant CXCL1, and F-actin was localized by phalloidin staining. CXCL1 induced an increase in F-actin formation over time. Stress fibers appeared rapidly and were most pronounced at cell-cell borders. After 120 minutes, most endothelial cells appeared in a polygonal, retracted shape. Images are representative of 3 experiments with similar results.

Reaction conditions were optimized, and cDNA samples were multiplexed with GAPDH and CXCR2 primers on an iCycler iQ Real-Time Detection System (Bio-Rad). Values were determined using iCycler iQ Real-Time Detection System software (version 3.0a; Bio-Rad) and normalized to GAPDH. The primers for CXCR2 were as follows: forward, AGCAAACACCTCTACTACCCTCTA; reverse, GGGCTGCATCAATTCAAATACCA; probe, ACCTAAACGGTGGCCCCTCGGGG. The primers for GAPDH were as follows: forward, GGCTCATGACCACAGTCCAT; reverse, GCCTGCTTCACCACCTTCT; probe, CCTGGAGAAACCTGCCAAGTATGATGAC.

Immunohistochemistry. $\mathrm{CXCR} 2^{+/+}$and $\mathrm{CXCR2^{-/- }}$ mice were euthanized, and the pulmonary circulation was perfused free of blood with $10 \mathrm{ml} \mathrm{PBS} \mathrm{(pH} \mathrm{7.4,}$ $4^{\circ} \mathrm{C}$ ) at $20 \mathrm{cmH}_{2} \mathrm{O}$ via the spontaneously beating right ventricle. The trachea was cannulated, and the lung was inflated with $4 \%$ paraformaldehyde (PFA) for 10 minutes at $25 \mathrm{cmH}_{2} \mathrm{O}$. The lungs were subsequently removed and fixed in PFA for 24 hours. Paraffin-embedded sections $(5 \mu \mathrm{m})$ were stained for CXCR2 using the avidin-biotin technique (Vector Laboratories) as previously described (45). Briefly, deparaffinized and rehydrated sections were incubated with avidin, $10 \%$ rabbit serum, and $0.5 \%$ fish skin gelatin oil for 1 hour to block nonspecific binding. After washing with PBS, rat anti-mouse CXCR2 was added $(1 \mu \mathrm{g} / \mathrm{ml})$ and incubated overnight. Sections were then washed and incubated with $5 \mu \mathrm{g} / \mathrm{ml}$ biotinylated rabbit anti-rat IgG (Vector Laboratories) for 1 hour, followed by avidin-biotin-peroxidase complexes (Vectastain Elite ABC kit; Vector Laboratories), washed with PBS, incubated with diaminobenzidine (DAB kit; Vector Laboratories) and counterstained with hematoxylin.

Pulmonary microvascular permeability. We determined pulmonary microvascular permeability in $\mathrm{CXCR2} 2^{++}, \mathrm{CXCR2} 2^{--}$, and chimeric mice using the Evans blue dye extravasation technique (46). Evans blue (20 mg/kg; Sigma-Aldrich) was injected intravenously 30 minutes prior to euthanasia. Lungs were perfused through the spontaneously beating right ventricle to remove intravascular dye. Lungs were removed, and Evans blue was extracted as described previously (47). The absorption of Evans blue was measured at $620 \mathrm{~nm}$ and corrected for the presence of heme pigments: $\mathrm{A}_{620}$ (corrected) $=\mathrm{A}_{620}-\left(1.426 \times \mathrm{A}_{740}+0.030\right)$ (48). Extravasated Evans blue was determined in the different animal groups 6 hours after LPS or saline inhalation and calculated against a standard curve (micrograms Evans blue dye per gram lung).

Cytoskeletal remodeling. To demonstrate that pulmonary endothelial cells are capable of responding to CXCR2-mediated activation, we investigated the distribution of F-actin as described previously $(35,49)$. Briefly, endothelial cells were allowed to adhere on gelatin-coated glass coverslips overnight (medium with $10 \% \mathrm{FBS}$ ). Cells were then serum-starved for 6 hours and treated with 10 or $100 \mathrm{ng} / \mathrm{ml}$ of recombinant CXCL1 (Peprotech). Untreated cells served as control. At different times, cells were washed, fixed (4\% PFA), permeabilized ( $0.1 \%$ Triton X-100, SigmaAldrich), and stained with FITC-phalloidin (Invitrogen Corp.). Coverslips were mounted on glass slides, and microscopy was performed on a Nikon Diaphot inverted fluorescence microscope.

Cytospins of BAL. Cytospins of BAL from CXCR2 $2^{+/+}$and CXCR2 $2^{-/-}$mice harvested 12 hours after LPS exposure were prepared using a cytocentrifuge (Thermo Shandon). Cytospun cells were stained (Diff-Quick staining; IMEB Inc.), air dried, and coverslipped.

Blood counts. To reveal possible differences among the different groups of mice, baseline differential blood counts were performed (Hemavet $850 \mathrm{FS}$; CDC Technologies Inc.).

Statistics. Statistical analysis was performed with JMP Statistical Software (version 5.1; SAS Institute Inc.). Differences between the groups were evaluated by 1 -way ANOVA followed by a post-hoc Tukey test. Data were presented as mean $\pm \mathrm{SD}$, and $P \leq 0.05$ was considered statistically significant.

\section{Acknowledgments}

The authors wish to thank Li-Ping Huang for help with immunohistochemistry and Rebecca Stockton for help with F-actin staining. This study was supported by the Deutsche Forschungsgemeinschaft (grant RE 1683/2-1; to J. Reutershan) and by NIH grant HL73361 (to K. Ley and J. Linden).

Received for publication September 30, 2005, and accepted in revised form December 13, 2005.

Address correspondence to: Klaus Ley, University of Virginia Health System, Cardiovascular Research Center, PO Box 801394, Charlottesville, Virginia 22908-1394, USA. Phone: (434) 243-9966; Fax: (434) 924-2828; E-mail: klausley@virginia.edu. 
1. Ware, L.B., and Matthay, M.A. 2000. The acute respiratory distress syndrome. N. Engl. J. Med. 342:1334-1349.

2. Abraham, E., Carmody, A., Shenkar, R., and Arcaroli, J. 2000. Neutrophils as early immunologic effectors in hemorrhage- or endotoxemia-induced acute lung injury. Am. J. Physiol. Lung Cell. Mol. Physiol. 279:L1137-L1145.

3. Azoulay, E., et al. 2002. Deterioration of previous acute lung injury during neutropenia recovery. Crit. Care Med. 30:781-786.

4. Reutershan, J., Basit, A., Galkina, E.V., and Ley, K. 2005. Sequential recruitment of neutrophils into lung and bronchoalveolar lavage fluid in LPSinduced acute lung injury. Am. J. Physiol. Lung Cell. Mol. Physiol. 289:L807-L815.

5. Doerschuk, C.M. 2001. Mechanisms of leukocyte sequestration in inflamed lungs. Microcirculation. 8:71-88.

6. Razavi, H.M., et al. 2004. Pulmonary neutrophil infiltration in murine sepsis: role of inducible nitric oxide synthase. Am. J. Respir. Crit. Care Med. 170:227-233.

7. Doerschuk, C.M. 2004. NO and neutrophils during sepsis: NO says "yes" to sequestration but "no" to migration. Am. J. Respir. Crit. Care Med. 170:205-206.

8. Olson, T.S., and Ley, K. 2002. Chemokines and chemokine receptors in leukocyte trafficking. Am. J. Physiol. Regul. Integr. Comp. Physiol. 283:R7-R28.

9. Kurdowska, A., et al. 2002. Anti-interleukin-8 autoantibodies in patients at risk for acute respiratory distress syndrome. Crit. Care Med. 30:2335-2337.

10. Kurdowska, A., et al. 2001. Anti-interleukin 8 autoantibody: interleukin 8 complexes in the acute respiratory distress syndrome. Relationship between the complexes and clinical disease activity. Am. J. Respir. Crit. Care Med. 163:463-468.

11. Matityahu, E., Feniger-Barish, R., Meshel, T. Zaslaver, A., and Ben Baruch, A. 2002. Intracellular trafficking of human CXCR1 and CXCR2: regulation by receptor domains and actin-related kinases. Eur. J. Immunol. 32:3525-3535.

12. Baggiolini, M., Dewald, B., and Moser, B. 1997. Human chemokines: an update. Annu. Rev. Immunol. 15:675-705.

13. Jones, S.A., Dewald, B., Clark-Lewis, I., and Baggiolini, M. 1997. Chemokine antagonists that discriminate between interleukin-8 receptors. Selective blockers of CXCR2. J. Biol. Chem. 272:16166-16169.

14. Casilli, F., et al. 2005. Inhibition of interleukin-8 (CXCL8/IL-8) responses by repertaxin, a new inhibitor of the chemokine receptors CXCR1 and CXCR2. Biochem. Pharmacol. 69:385-394.

15. Hillyer, P., Mordelet, E., Flynn, G., and Male, D. 2003. Chemokines, chemokine receptors and adhesion molecules on different human endothelia: discriminating the tissue-specific functions that affect leucocyte migration. Clin. Exp. Immunol. 134:431-441.

16. Keane, M.P., Belperio, J.A., Xue, Y.Y., Burdick, M.D. and Strieter, R.M. 2004. Depletion of CXCR2 inhibits tumor growth and angiogenesis in a murine model of lung cancer. J. Immunol. 172:2853-2860.

17. Belperio, J.A., et al. 2005. Role of CXCR2/CXCR2 ligands in vascular remodeling during bronchiolitis obliterans syndrome. J. Clin. Invest. 115:1150-1162. doi:10.1172/JCI200524233.

18. Sue, R.D., et al. 2004. CXCR2 is critical to hyperoxiainduced lung injury. J. Immunol. 172:3860-3868.

19. Lomas-Neira, J.L., Chung, C.S., Grutkoski, P.S., Miller, E.J., and Ayala, A. 2004. CXCR2 inhibition suppresses hemorrhage-induced priming for acute lung injury in mice. J. Leukoc. Biol. 76:58-64.

20. Johnston, R.A., Mizgerd, J.P., and Shore, S.A 2005. CXCR2 is essential for maximal neutrophil recruitment and methacholine responsiveness after ozone exposure. Am. J. Physiol. Lung Cell. Mol. Physiol. 288:L61-L67.

21. Belperio, J.A., et al. 2002. Critical role for CXCR2 and CXCR2 ligands during the pathogenesis of ventilator-induced lung injury. J. Clin. Invest. 110:1703-1716. doi:10.1172/JCI200215849.

22. Cacalano, G., et al. 1994. Neutrophil and B cell expansion in mice that lack the murine IL-8 receptor homolog. Science. 265:682-684.

23. Doerschuk, C.M., et al. 1987. Marginated pool of neutrophils in rabbit lungs. J. Appl. Physiol. 63:1806-1815.

24. [Anonymous]. 2000. Ventilation with lower tidal volumes as compared with traditional tidal volumes for acute lung injury and the acute respiratory distress syndrome. The Acute Respiratory Distress Syndrome Network. N. Engl. J. Med. 342:1301-1308.

25. Reutershan, J., and Ley, K. 2004. Bench-to-bedside review: Acute respiratory distress syndrome how neutrophils migrate into the lung. Crit. Care. 8:453-461.

26. Burns, J.A., Issekutz, T.B., Yagita, H., and Issekutz, A.C. 2001. The alpha 4 beta 1 (very late antigen (VLA)-4, CD49d/CD29) and alpha 5 beta 1 (VLA-5 CD49e/CD29) integrins mediate beta 2 (CD11/ CD18) integrin-independent neutrophil recruitment to endotoxin-induced lung inflammation. J. Immunol. 166:4644-4649.

27. Mizgerd, J.P., Horwitz, B.H., Quillen, H.C., Scott, M.L., and Doerschuk, C.M. 1999. Effects of CD18 deficiency on the emigration of murine neutrophils during pneumonia. J. Immunol. 163:995-999.

28. Fu, W., Zhang, Y., Zhang, J., and Chen, W.F. 2005. Cloning and characterization of mouse homolog of the CXC chemokine receptor CXCR1. Cytokine. 31:9-17.

29. Moepps, B., Nuesseler, E., Braun, M., and Gierschik, P. 2005. A homolog of the human chemokine receptor CXCR1 is expressed in the mouse. Mol. Immunol. 43:897-914.

30. Vanderbilt, J.N., et al. 2003. CXC chemokines and their receptors are expressed in type II cells and upregulated following lung injury. Am. J. Respir. Cell Mol. Biol. 29:661-668.

31. Souza, D.G., et al. 2004. Repertaxin, a novel inhibitor of rat CXCR2 function, inhibits inflammatory responses that follow intestinal ischaemia and reperfusion injury. Br. J. Pharmacol. 143:132-142.

32. Liehn, E.A., Schober, A., and Weber, C. 2004. Blockade of keratinocyte-derived chemokine inhibits endothelial recovery and enhances plaque formation after arterial injury in ApoE-deficient mice. Arterioscler. Thromb. Vasc. Biol. 24:1891-1896.

33. Middleton, J., et al. 1997. Transcytosis and surface presentation of IL-8 by venular endothelial cells. Cell. 91:385-395.

34. Worthen, G.S., Schwab, B., III, Elson, E.L., and Downey, G.P. 1989. Mechanics of stimulated neutrophils: cell stiffening induces retention in capillaries. Science. 245:183-186.

35. Schraufstatter, I.U., Chung, J., and Burger, M. 2001 IL-8 activates endothelial cell CXCR1 and CXCR2 through Rho and Rac signaling pathways. Am. J. Physiol. Lung Cell. Mol. Physiol. 280:L1094-L1103.

36. MacCallum, N.S., and Evans, T.W. 2005. Epidemiology of acute lung injury. Curr. Opin. Crit. Care. 11:43-49.

37. Boe, D.M., Nelson, S., Zhang, P., and Bagby, G.J. 2001. Acute ethanol intoxication suppresses lung chemokine production following infection with Streptococcus pneumoniae. J. Infect. Dis. 184:1134-1142.

38. Ness, T.L., Hogaboam, C.M., Strieter, R.M., and Kunkel, S.L. 2003. Immunomodulatory role of CXCR2 during experimental septic peritonitis. J. Immunol. 171:3775-3784.

39. Kaneider, N.C., Agarwal, A., Leger, A.J., and Kuliopulos, A. 2005. Reversing systemic inflammatory response syndrome with chemokine receptor pepducins. Nat. Med. 11:661-665.

40. Jin, Q., et al. 2004. Discovery of potent and orally bioavailable N,N'-diarylurea antagonists for the CXCR2 chemokine receptor. Bioorg. Med. Chem. Lett. 14:4375-4378.

41. Widdowson, K.L., et al. 2004. Evaluation of potent and selective small-molecule antagonists for the CXCR2 chemokine receptor. J. Med. Chem. 47:1319-1321.

42. Forlow, S.B., et al. 2001. Increased granulopoiesis through interleukin-17 and granulocyte colonystimulating factor in leukocyte adhesion moleculedeficient mice. Blood. 98:3309-3314.

43. Suratt, B.T., et al. 2001. Neutrophil maturation and activation determine anatomic site of clearance from circulation. Am. J. Physiol. Lung Cell. Mol. Physiol. 281:L913-L921.

44. Boe, D.M., Nelson, S., Zhang, P., Quinton, L., and Bagby, G.J. 2003. Alcohol-induced suppression of lung chemokine production and the host defense response to Streptococcus pneumoniae. Alcobol Clin. Exp. Res. 27:1838-1845

45. Olson, T.S., Singbartl, K., and Ley, K. 2002. L-selectin is required for fMLP- but not C5a-induced margination of neutrophils in pulmonary circulation. Am.J. Physiol Regul. Integr. Comp. Physiol. 282:R1245-R1252.

46. Green, T.P., Johnson, D.E., Marchessault, R.P., and Gatto, C.W. 1988. Transvascular flux and tissue accrual of Evans blue: effects of endotoxin and histamine. J. Lab. Clin. Med. 111:173-183.

47. Peng, X., et al. 2004. Protective Effects of Sphingosine 1-Phosphate in murine endotoxin-induced inflammatory lung injury. Am. J. Respir. Crit. Care Med. 169:1245-1251.

48. Wang, L.F., et al. 2002. Role of inducible nitric oxide synthase in pulmonary microvascular protein leak in murine sepsis. Am. J. Respir. Crit. Care Med. 165:1634-1639.

49. Stockton, R.A., Schaefer, E., and Schwartz, M.A. 2004. p21-activated kinase regulates endothelial permeability through modulation of contractility. J. Biol. Chem. 279:46621-46630. 